\title{
Effects and safety of rituximab in systemic sclerosis: an analysis from the European Scleroderma Trial and Research (EUSTAR) group
}

\author{
Suzana Jordan, ${ }^{1}$ Jörg H W Distler, ${ }^{2}$ Britta Maurer, ${ }^{1}$ Dörte Huscher, ${ }^{3}$ \\ Jacob M van Laar, ${ }^{4}$ Yannick Allanore, ${ }^{5}$ Oliver Distler, ${ }^{1}$ on behalf of the EUSTAR \\ Rituximab study group
}

\begin{abstract}
Handling editor Tore K Kvien
- Additional material is published online only. To view please visit the journal online (http://dx.doi.org/10.1136/ annrheumdis-2013-204522).

1 Divison of Rheumatology, University Hospital Zurich, Zurich, Switzerland ${ }^{2}$ Department of Internal Medicine, University of Erlangen-Nuremberg, Erlangen, Germany

${ }^{3}$ Charité University Hospital and German Rheumatism Research Centre, Berlin, Germany

${ }^{4}$ Department of Rheumatology and Clinical Immunology UMC, Utrecht, The Netherlands ${ }^{5}$ Université Paris Descartes, Hôpital Cochin, Service de Rhumatologie A \& INSERM U1016, Paris, France
\end{abstract}

\section{Correspondence to} Dr Oliver Distler, Division of Rheumatology, University Hospital Zurich, Gloriastr. 25; Zurich 8091 , Switzerland; Oliver.Distler@usz. ch

YA and OD contributed equally.

Received 29 August 2013 Revised 12 December 2013 Accepted 27 December 2013 Published Online First 17 January 2014

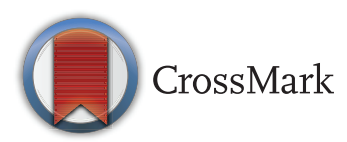

To cite: Jordan $\mathrm{S}$, Distler JHW, Maurer B, et al. Ann Rheum Dis

2015;74:1188-1194.

\section{ABSTRACT}

Objectives To assess the effects of Rituximab (RTX) on skin and lung fibrosis in patients with systemic sclerosis (SSC) belonging to the European Scleroderma Trial and Research (EUSTAR) cohort and using a nested case-control design.

Methods Inclusion criteria were fulfilment of American College of Rheumatology classification criteria for SSc, treatment with RTX and availability of follow-up data. RTX-treated patients were matched with control patients from the EUSTAR database not treated with RTX. Matching parameters for skin/lung fibrosis were the modified Rodnan Skin Score (mRSS), forced vital capacity $(\mathrm{FVC})$, follow-up duration, scleroderma subtype, disease duration and immunosuppressive co-treatment. The primary analysis was $\mathrm{mRSS}$ change from baseline to follow-up in the RTX group compared with the control group. Secondary analyses included change of FVC and safety measures.

Results 63 patients treated with RTX were included in the analysis. The case-control analysis in patients with severe diffuse SSc showed that mRSS changes were larger in the RTX group versus matched controls $(\mathrm{N}=25$; $-24.0 \pm 5.2 \%$ vs $-7.7 \pm 4.3 \% ; p=0.03$ ). Moreover, in RTX-treated patients, the mean mRSS was significantly reduced at follow-up compared with baseline $(26.6 \pm 1.4$ vs $20.3 \pm 1.8 ; p=0.0001)$. In addition, in patients with interstitial lung disease, RTX prevented significantly the further decline of FVC compared with matched controls $(\mathrm{N}=9 ; 0.4 \pm 4.4 \%$ vs $-7.7 \pm 3.6 \% ; p=0.02)$. Safety measures showed a good profile consistent with previous studies in autoimmune rheumatic diseases.

Conclusions The comparison of RTX treated versus untreated matched-control SSc patients from the EUSTAR cohort demonstrated improvement of skin fibrosis and prevention of worsening lung fibrosis, supporting the therapeutic concept of B cell inhibition in SSC.

\section{INTRODUCTION}

There is a strong rationale behind the use of $\mathrm{B}$ cell depletion in the treatment of systemic sclerosis (SSc), a life-threatening, connective tissue disease, characterised by vasculopathy, fibrosis and autoimmunity. Abnormalities of B cell function have been demonstrated in both animal models of SSc and in SSc patients. B cell infiltration and gene expression of B cell-related genes were found in skin biopsies from SSc patients and in lungs of patients with SSc-associated interstitial lung disease (SSc-ILD). ${ }^{1-3}$ Major positive (CD19) and negative (CD22) regulators of $\mathrm{B}$ cell response were found to be dysbalanced in SSc patients and murine SSc. ${ }^{4-9}$ Moreover, B cell homeostasis has been shown to be disrupted in SSc in favour of naive $B$ cells. Although diminished, remaining memory $\mathrm{B}$ cells are hyper-reactive and showed enhanced capabilities to produce immunoglobulin and autoantibodies. ${ }^{10}$ Augmented $\mathrm{B}$ cell activating factor signalling in SSc and murine models leads to overproduction of cytokines that promote fibrosis. ${ }^{11} 12$ Activated $\mathrm{B}$ cells regulate $\mathrm{T}$ cell activation and differentiation by promoting type $2 \mathrm{~T}$ helper cells, shifting cytokine production towards the profibrotic cytokines interleukin (IL)-6, IL-4 and IL-13. ${ }^{13}$ Besides, $\mathrm{B}$ cells produce transforming growth factor $\beta$, a strong profibrotic stimulus. ${ }^{14}{ }^{15}$ Moreover, B cell depletion in tight skin mice-1 reduced skin fibrosis and autoimmunity. ${ }^{16}$ Taken together, there is ample evidence of B cell involvement in the pathogenesis of fibrosis in SSc, making it a promising potential target for treatment of skin and lung fibrosis in SSc.

Rituximab (RTX) is a chimeric monoclonal antibody that targets CD20, which is expressed from pre-B cell stage to the preplasma cell stage. It was originally approved for the treatment of non-Hodgkin's lymphoma and later for the treatment of rheumatoid arthritis and ANCA-associated vasculitis. Evidence for potential efficacy of RTX in other autoimmune diseases is growing. ${ }^{17}$

Currently, there are case reports ${ }^{18-20}$ and a few open-labelled, uncontrolled studies on RTX in the treatment of SSc. ${ }^{121-24}$ Although there is inconsistency due to different study designs in these data, there is evidence of an antifibrotic effect of RTX. In all case reports, there was an improvement in lung function and dyspnoea functional class. ${ }^{18-20}$ In one case, beneficial effects on skin fibrosis were shown, ${ }^{19}$ while in the other cases effect of RTX on skin fibrosis was not reported..$^{18}{ }^{20}$ In one smaller single centre, randomised controlled study with eight RTX treated and six controls and in a long-term RTX treatment, skin and lung function improved, as well as overall function as measured by the Health Assessment Questionnaire Disability Index score. ${ }^{21} 25-28$ Bosello et $a l^{22}$ and Smith $e t a l^{23}$ reported skin improvement after RTX treatment. ${ }^{24}$ Lafyatis et al did not find 
beneficial effects on skin disease and lung function of SSc patients versus baseline, but reported a complete depletion of B cell infiltrates in skin biopsies and good safety and tolerance of RTX. ${ }^{1}$ While these studies provide important insights on the use of RTX in SSc, they are limited by the low number of patients, the lack of a sufficient control arm and the single centre design. To address these limitations, our objective was to perform a multicentre, nested case-control study to analyse effects of RTX on skin and lung fibrosis and safety in a real-life clinical setting using the European Scleroderma Trial and Research (EUSTAR) cohort.

\section{METHODS}

\section{Study design}

In all, 42 EUSTAR centres participated in this multicentre, post hoc nested case control, observational study. Data about RTX-treated SSc patients were collected from centres during a 6-month period. Patients were prospectively included in the EUSTAR database and received RTX in routine clinical practice upon the decision of their physicians. Patients for the matchedcontrol group were chosen from the EUSTAR database according to the matching criteria outlined below and consisted of SSc patients not treated with RTX. Clinical characteristics of patients and outcome parameters were defined as in the EUSTAR online Minimal Essential Data Set and have been reported in detail. ${ }^{29}{ }^{30}$ Additional data with a special focus on timing of RTX exposure and immunosuppressive co-treatment, immune profile and safety issues were provided retrospectively by the participating centres. Immunosuppressive co-treatment was defined as ongoing or newly started treatment at RTX treatment including a 3-month period before the first RTX infusion.

The following clinical data were included into the analysis: Patients' demographics (age, gender), disease duration from the first non-Raynaud's symptom, disease subtype (limited or diffuse SSc) and autoantibodies status (presence of anticentromere, anti-RNA polymerase III, antitopoisomerase 1 (anti-Scl-70), anti-U1 small nuclear ribonucleoprotein). Skin fibrosis was assessed by the modified Rodnan Skin Score (mRSS). ${ }^{31}$ EUSTAR centres are advised that the same investigator is performing the mRSS on follow-up visits in individual

Table 1 Baseline demographics and clinical characteristics of all analysed SSC patients $(\mathrm{N}=63)$

\begin{tabular}{|c|c|c|}
\hline \multicolumn{3}{|l|}{ Baseline characteristics $(\mathrm{N}=63)$} \\
\hline Age (years) mean \pm SEM & \multicolumn{2}{|c|}{$\mathrm{N}=63 ; 50.9 \pm 1.6$} \\
\hline Sex & $\mathrm{n}$ & $(\%)$ \\
\hline Female & 45 & 71.4 \\
\hline \multicolumn{3}{|l|}{ Scleroderma subtype } \\
\hline Diffuse & 46 & 73.0 \\
\hline Limited & 17 & 27.0 \\
\hline Disease duration in years median (IQR) & $\mathrm{N}=60$ & \\
\hline Follow-up in months (IQR) & $7(4-9)$ & \\
\hline Autoantibodies positive & $\mathrm{n} / \mathrm{N}$ & $(\%)$ \\
\hline ACA & $3 / 62$ & 4.8 \\
\hline Anti-RNA polymerase III & $3 / 52$ & 5.8 \\
\hline Anti-Scl-70 & $42 / 61$ & 68.9 \\
\hline Anti-U1-snRNP & $2 / 50$ & 4.0 \\
\hline \multicolumn{3}{|c|}{$\begin{array}{l}\text { Autoantibodies were measured and interpreted according to local standards. } \\
\text { Demographics and clinical characteristics are defined according to EUSTAR criteria. }{ }^{30} \\
\text { ACA, anticentromere antibody; anti-Scl 70, antitopoisomerase } 1 \text { antibody; } \\
\text { anti-U1-snRNP, anti-U1 small nuclear ribonucleoprotein antibody; EUSTAR, European } \\
\text { Scleroderma Trial and Research; N, number of patients available for analysis; } \\
\text { n, number of patients with the specific feature; SSc, systemic sclerosis. }\end{array}$} \\
\hline
\end{tabular}

patients, and EUSTAR investigators are trained on a regular basis on how to perform the mRSS. ${ }^{32}{ }^{33}$ ILD was assessed by lung function tests including forced vital capacity (FVC) and diffusing capacity of the lung for carbon monoxide (DLCO), and by high-resolution CT (HRCT). ${ }^{22}$

All patients agreed to participate in the EUSTAR database by signing informed consent forms approved by the local ethical committees. The study was conducted in accordance with the principles of the Declaration of Helsinki and local laws and guidelines for Good Clinical Practice.

\section{Inclusion and exclusion criteria}

Patients were included in the RTX group if they met the American College of Rheumatology (ACR) criteria for $\mathrm{SSc}^{34}$ were treated with RTX and had at least one follow-up. Patients in the matched-control group had to fulfil the ACR criteria for SSc, ${ }^{34}$ did not receive RTX treatment and had at least one follow-up with report of the mRSS. Patients were excluded if they had autologous stem cell transplantation between baseline and follow-up. For the analysis of RTX on SSc-ILD, we included SSc patients with evidence for ILD, as defined by FVC of less than $70 \%$ predicted and evidence for lung fibrosis on HRCT at baseline.

\section{Primary and secondary objectives}

The primary objective for this study was to measure the change of mRSS from baseline to follow-up between the RTX and control groups. Secondary objectives were to measure the change of the FVC from baseline to follow-up between the RTX and control groups in patients with evidence for ILD and safety measures. Effects of RTX on primary and secondary objectives were analysed at the first follow-up after a single RTX course.

\section{Statistical analysis and matching}

RTX-treated patients were matched with control patients from the EUSTAR database. Matching parameters for skin fibrosis were: $\operatorname{mRSS}(\max \pm 25 \%)$, follow-up duration ( $\max \pm 3$ months), scleroderma subtype (diffuse or limited), disease duration (best match in years) and immunosuppressive co-treatment (including $\geq 10 \mathrm{mg}$ /day prednisone). Matching parameters for ILD were: baseline FVC ( $\max \pm 10 \%)$, follow-up duration ( $\max \pm 3$ months), disease duration (best match in years) and immunosuppressive co-treatment. Subanalysis was performed for patients with severe diffuse $S S c$ as defined by $m R S S \geq 16 / 51$.

Statistical analysis was performed using GraphPad Prism V.5 software and a single sample test on mean of 0 by SPSS software. Normal distribution was analysed by the d'Agostino and Pearson omnibus normality test. Normally distributed data are shown as mean \pm SEM and were analysed by paired $t$ test. Non-parametric data including the mRSS are shown as median and IQR and were analysed by Wilcoxon matched paired signed rank test. Categorical variables were analysed by Fisher's exact test. Percental change between baseline and follow-up was calculated by single sample test on mean of 0 . $p$ Values $\leq 0.05$ were considered statistically significant.

\section{RESULTS}

\section{Baseline}

In all, 42 EUSTAR centres contributed to this study and data from 72 SSc patients with RTX therapy were collected. One patient was excluded due to an interfering therapy (autologous stem cell transplantation) between baseline and follow-up and one did not fulfil ACR criteria. Seven patients were excluded 
because the follow-up visit was not available. Baseline demographics and clinical characteristics of the remaining $63 \mathrm{SSc}$ patients included in the study are shown in table 1.

As expected for patients who received RTX on the decision of their treating physician in routine practice, patients were enriched for diffuse SSc $(n=35 / 63)$.

\section{RTX application scheme}

We analysed the effects after one course of RTX. The most frequent application was two infusions of $1000 \mathrm{mg}$ in 2 weeks (in $75 \%$ of patients), but there were also other application schemes (table 2). Overall, 31 patients (31/63 or 49\%) received co-treatment with methylprednisolone during RTX application, all of them in a dose of $100 \mathrm{mg}$ per infusion according to the general recommendations for RTX pretreatment. A total of 41 patients $(65 \%)$ received co-treatment with disease modifying antirheumatic drugs (DMARDs) (table 2).

\section{Effects on skin fibrosis}

We started the analysis with the whole group of patients including patients with limited SSc. There were 46/63 SSc patients who had mRSS reported at baseline and follow-up available for this analysis. Disease duration was $5(3-10)$ years, and 35 had diffuse SSc and 11 limited SSc. After a follow-up of 7 (5-9) months, the mRSS decreased significantly from $18.1 \pm 1.6$ to $14.4 \pm 1.5$ $(p=0.0002)$ (figure 1A). Percentage change of mRSS versus baseline in this group was $-15.0 \pm 5.3 \%(\mathrm{p}=0.008)$ (figure $1 \mathrm{~B})$.

Patients with limited SSc are not an optimal study population for clinical trials on skin fibrosis and their inclusion might mask

Table 2 Rituximab (RTX) application schemes and co-treatment with potentially disease modifying antirheumatic drugs (DMARDs)

\begin{tabular}{lrr}
\hline RTX application and co-treatment (N=63) & $\mathrm{n} / \mathrm{N}$ & $\mathbf{( \% )}$ \\
\hline RTX application (each administration within 2 weeks) & & \\
$1000 \mathrm{mg}$ & $13 / 63$ & 20.6 \\
$1000 \mathrm{mg} \times 2$ & $47 / 63$ & 74.6 \\
$1100 \mathrm{mg} \times 2$ & $1 / 63$ & 1.6 \\
$1295 \mathrm{mg} \times 2$ & $1 / 63$ & 1.6 \\
$500 \mathrm{mg} \times 6$ & $1 / 63$ & 1.6 \\
Co-treatment with DMARDs & & \\
Received one DMARD & $32 / 63$ & 50.8 \\
Methotrexate (MTX) & $13 / 58$ & 22.4 \\
Azathioprine (AZA) & $6 / 58$ & 10.3 \\
Prednisone $\geq 10 \mathrm{mg} /$ day & $5 / 61$ & 8.1 \\
Mycophenolate mofetil (MMF) & $4 / 57$ & 7.0 \\
Cyclophosphamide (CYC) & $1 / 57$ & 1.7 \\
Sulfasalazine & $1 / 48$ & 2.1 \\
Leflunomide & $1 / 48$ & 2.1 \\
Colchicine & $1 / 57$ & 1.8 \\
Received two DMARDs & $8 / 63$ & 12.7 \\
Prednisone $\geq 10 \mathrm{mg} /$ day+CYC & $2 / 57$ & 3.5 \\
Prednisone $\geq 10 \mathrm{mg} /$ day+AZA & $1 / 58$ & 1.7 \\
Prednisone $\geq 10 \mathrm{mg} /$ day+MTX & $1 / 58$ & 1.7 \\
AZA+hydroxychloroquine & $1 / 49$ & 2.0 \\
AZA+tacrolimus & $1 / 58$ & 1.7 \\
MTX+TNF- $\alpha$ inhibitors & $1 / 49$ & 2.0 \\
CYC+MMF & $1 / 57$ & 1.7 \\
Received three DMARDs & $1 / 63$ & 1.6 \\
Prednisone $\geq 10 \mathrm{mg} /$ day+MTX+cyclosporine (CSA) & $1 / 58$ & 1.7 \\
\hline P & & \\
\hline
\end{tabular}

Prednisone $\geq 10 \mathrm{mg} /$ day has been defined as DMARD.

$\mathrm{N}$, number of patients available for analysis; $n$, number of patients with the specific feature; TNF- $\alpha$, tumour necrosis factor $\alpha$. real clinical effects. Therefore, we focused next our analysis on patients with diffuse SSc $(n=35)$ who had a disease duration of 5 (3-6) years. Those patients showed a significant decrease in mean mRSS from $22.1 \pm 1.6$ to $17.7 \pm 1.6(\mathrm{p}=0.0005)$ after 6 (3-9) months follow-up (figure 1C). Percentage change of mRSS versus baseline was $-16.7 \pm 5.5 \%(p=0.005)$ (figure 1D).

These effects became even more evident when we enriched the population for severe, diffuse SSc patients (mRSS $\geq 16$ ), who are the standard population to be included in clinical trials on skin fibrosis. In these patients $(n=25)$, the mean mRSS decreased from $26.6 \pm 1.4$ to $20.3 \pm 1.8 \quad(p=0.0001)$ after 6 (5-9) months follow-up (figure 1E). Disease duration in this subgroup of patients with severe diffuse SSc was 5 (3-7) years. Percentage change of mRSS versus baseline was $-24.0 \pm 5.2 \%$ $(p=0.0001)$ (figure 1F).

Uncontrolled trials in SSc skin fibrosis are difficult to interpret because of the spontaneous decrease of the mRSS over time particularly in severe diffuse SSc. We therefore compared the patients with severe diffuse SSc treated with RTX with control patients from the EUSTAR database not treated with RTX. Controls were matched as described in the Methods section. Baseline characteristics of patients with severe diffuse SSc and matched controls are shown in table 3.

The results from this analysis further supported the effects of RTX on skin fibrosis. Patients under treatment with RTX showed a significantly lower mRSS at follow-up than matchedcontrol patients without RTX treatment $(n=25$ for each group, figure $2 \mathrm{~A}$ ). This referred to an absolute change from baseline of $6.3 \pm 1.4$ in the RTX group versus $-1.9 \pm 1.0$ in the control group $(p=0.02)$ (figure $2 \mathrm{~B})$. The percentage change in the RTX group versus baseline was above the minimal clinically important difference for the mRSS $(-24.0 \pm 5.2 \% \mathrm{vs}-7.7 \pm 4.3 \%$ in the control group; $\mathrm{p}=0.03$; figure $2 \mathrm{C}) .^{35}$

\section{Effects on lung fibrosis}

Effects of RTX treatment on lung function were analysed in SSc patients with FVC $<70 \%$ predicted, and with parallel evidence for ILD on HRCT $(n=9)$. The median disease duration in these patients was 6 (4-12) years, $6(66 \%)$ had diffuse SSc, mean FVC \% predicted was $60.6 \pm 2.4$ and mean DLCO \% predicted was 41.1 \pm 2.8 (baseline characteristics see online supplementary table S1).

Patients were analysed after median follow-up of 6 (4-12) months. In these patients with SSc-ILD, FVC was stable after RTX treatment compared with baseline $(60.6 \pm 2.4$ vs 61.3 $\pm 4.1 \% ; \mathrm{p}=0.5$ ). The DLCO was significantly improved in patients treated with RTX compared with baseline $(41.1 \pm 2.8$ vs $44.8 \pm 2.7 \% ; \mathrm{p}=0.03)$.

As in the analysis for skin fibrosis, we next matched RTX-treated patients with control patients without RTX treatment from the EUSTAR database (baseline characteristics see online supplementary table S1). In contrast to RTX-treated patients, matched controls showed a decline in FVC at follow-up (figure 3A). This resulted in significant differences between RTX treated and matched controls in change of FVC\% predicted in both the percentage $(0.4 \pm 4.4$ vs $-7.7 \pm 3.6$; $\mathrm{p}=0.02)$ and the absolute change $(0.8 \pm 2.2$ vs $-4.8 \pm 1.7$; $\mathrm{p}=0.01$ ) (figure $3 \mathrm{~B}$ ). There was no significant difference in change of DLCO between RTX-treated and matched-control patients $(3.7 \pm 1.4$ vs $6.2 \pm 6.2 ; \mathrm{p}=0.9)$.

\section{Safety of RTX}

Data on all 63 patients were available for safety analysis. We observed the following adverse events in RTX-treated patients: cardiac/renal involvement and arrhythmia was reported in one 
Figure 1 Change of modified Rodnan Skin Score (mRSS) after rituximab treatment in the whole available cohort $(\mathrm{N}=46)$ including patients with limited systemic sclerosis (SSC) (A and B), in diffuse SSc patients $(\mathrm{N}=35 ; \mathrm{C}$ and $\mathrm{D})$ and in diffuse severe SSc patients (mRSS $>16$ at baseline, $\mathrm{N}=25$ ) ( $\mathrm{E}$ and $\mathrm{F}$ ). ( $\mathrm{A}, \mathrm{C}$ and $\mathrm{E})$ Absolute mRSS values; $(B, D$ and $F)$ percental changes from baseline.

\section{Whole Cohort}

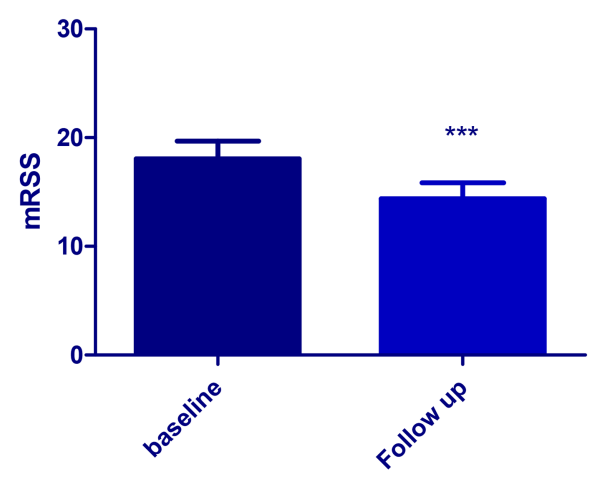

$\mathrm{N}=46 ; 18.1+1.6$ vs. $14.4+1.5 ; p=0.0002$

A

Diffuse SSc

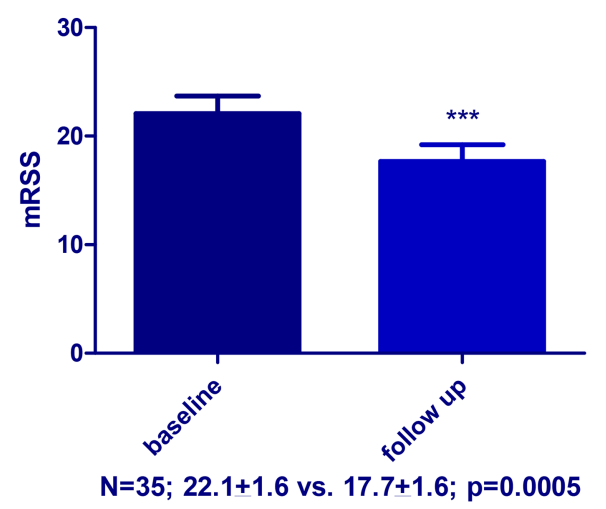

$\mathrm{C}$

Severe diffuse SSc

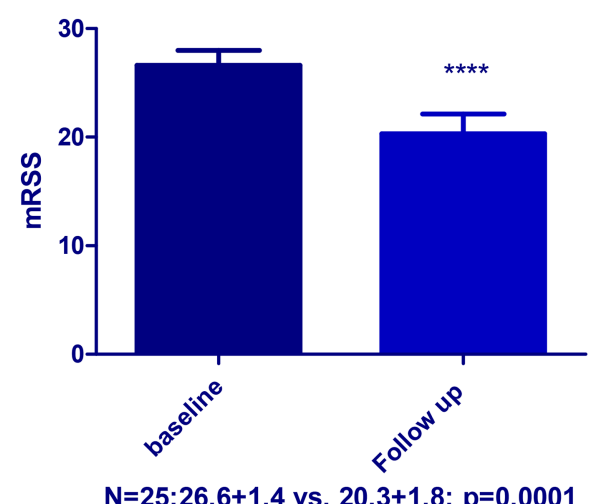

E

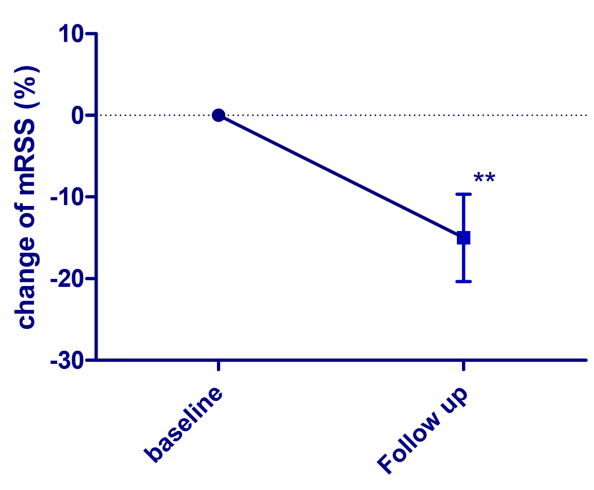

$N=46 ; 0$ vs. $-15.0+5.3 \% ; p=0.008$

B

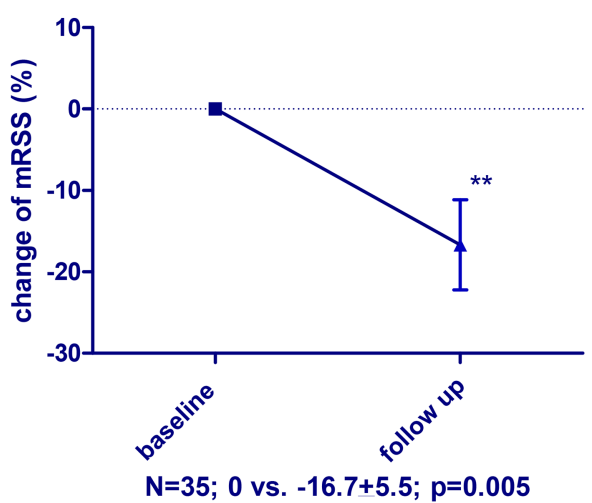

D

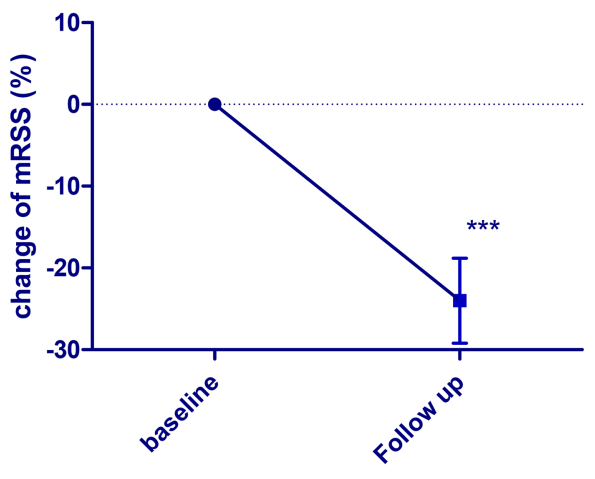

$n=25 ; 0$ vs. $-24.0+5.2 \% ; p=0.0001$

F patient each. Fatigue was noted in 14/56 (25\%), infections (as defined by experts) in $11 / 53(21 \%)$, nausea in $2 / 48(4 \%)$ and rigour in $3 / 48(6 \%)$. Serum sickness/hypersensitivity reaction was observed in 2/54 (4\%) patients. Both patients received co-treatment with MTX (15 and $20 \mathrm{mg} /$ week, respectively). No renal crisis was reported despite the frequent use of methylprednisolone during RTX application. Serious adverse events were not reported.

\section{DISCUSSION}

This is the first multicentre nested case-controlled study with RTX in SSc. Although there is a potential selection bias in this study, treatment with RTX improved skin fibrosis and stabilised lung function parameters in patients with SSc-associated lung fibrosis. It is noteworthy that effects of RTX could be shown in both skin and lung fibrosis. These effects on two different organ manifestations of fibrosis underline the potential of RTX as an antifibrotic agent in SSc. The most important advantage over previous studies on RTX in SSc was the inclusion of a large control group using a nested case-control design. Inclusion of a matched-control group is of particular importance in studies on skin fibrosis in SSc, as spontaneous improvements of the mRSS over time occur frequently. Thus, previous reported effects of RTX in uncontrolled studies might have been due to the natural 
Table 3 Baseline demographics and clinical characteristics of diffuse, severe SSc patients treated with RTX matched with diffuse, severe control SSC patients ( $\mathrm{N}=25$ pairs)

\begin{tabular}{|c|c|c|c|c|c|}
\hline \multicolumn{6}{|c|}{ Baseline characteristics of SSC patients with diffuse severe skin fibrosis } \\
\hline & \multicolumn{2}{|c|}{ RTX treated } & \multicolumn{2}{|c|}{$\begin{array}{l}\text { Matched } \\
\text { control }\end{array}$} & $\mathrm{p}$ Value \\
\hline Age (years) mean \pm SEM & \multicolumn{2}{|c|}{$\mathrm{N}=25 ; 45.0 \pm 2.4$} & \multicolumn{2}{|c|}{$\mathrm{N}=25 ; 50.0 \pm 3.0$} & 0.2 \\
\hline Sex & $\mathrm{n} / \mathrm{N}$ & $\%$ & $\mathrm{n} / \mathrm{N}$ & $\%$ & \\
\hline female & 16 & 64.0 & 19 & 76.0 & 0.5 \\
\hline mRSS & \multicolumn{2}{|c|}{$N=25 ; 26.6 \pm 1.4$} & \multicolumn{2}{|c|}{$\mathrm{N}=25 ; 25.0 \pm 1.2$} & $0.03^{*}$ \\
\hline $\begin{array}{l}\text { Disease duration in years } \\
\text { median (range) }\end{array}$ & \multicolumn{2}{|c|}{$5(3-7)$} & \multicolumn{2}{|c|}{$5(3-7)$} & 0.9 \\
\hline Follow-up in months (range) & \multicolumn{2}{|c|}{$6(5-9)$} & \multicolumn{2}{|c|}{$7(4-9)$} & 0.4 \\
\hline Autoantibodies positive & $19 / 25$ & 76.0 & $15 / 20$ & 75.0 & 1.0 \\
\hline ACA & $1 / 25$ & 4.0 & $1 / 18$ & 5.5 & 1.0 \\
\hline Anti-RNA polymerase III & $3 / 21$ & 14.3 & $1 / 11$ & 9.1 & 1.0 \\
\hline Anti-Scl-70 & $16 / 23$ & 69.6 & $14 / 20$ & 70.0 & 1.0 \\
\hline Anti-U1-snRNP & $0 / 22$ & 0 & $1 / 15$ & 6.7 & 0.4 \\
\hline DMARDs treatment & $20 / 24$ & 83.3 & $16 / 22$ & 72.7 & 0.5 \\
\hline \multicolumn{6}{|c|}{$\begin{array}{l}\text { Autoantibodies were measured and interpreted according to local standards. } \\
\text { Demographics and clinical characteristics are defined according to EUSTAR criteria. }{ }^{30} \\
\text { ACA, anticentromere antibody; anti-Scl 70, antitopoisomerase } 1 \text { antibody; } \\
\text { anti-U1-snRNP, anti-U1 small nuclear ribonucleoprotein antibody; DMARDs, disease } \\
\text { modifying antirheumatic drugs; EUSTAR, European Scleroderma Trial and Research; } \\
\text { mRSS, modified Rodnan Skin Score; N, number of patients available for analysis; } \\
\text { n, number of patients with the specific feature; RTX, rituximab; SSc, systemic sclerosis. } \\
{ }^{*} p<0.05 \text {. }\end{array}$} \\
\hline
\end{tabular}

course of the disease rather than due to effects of RTX effects. Moreover, it is the largest study reported so far on the use of RTX in SSc. It also reflects clinical practice rather than potentially artificial clinical study situations, as patients and controls were derived from the observational EUSTAR registry and were treated by decisions of the local physician.

We could also observe an acceptable safety profile of RTX. Infections and respiratory tract infections in particular are one of the frequent side effects of RTX treatment. In studies with rheumatoid arthritis, respiratory tract infection occurred in 4\%9\% of RTX patients. However, patients receiving placebo showed similar or even higher infection rates, indicating that such adverse events could be associated with DMARD co-treatment or the natural disease course. ${ }^{36-38}$ In the present study, the side effect profile was in general consistent with recent reports in other rheumatic autoimmune diseases. The exception was rigour that occurred in three patients in our study and is neither a common manifestation of SSc nor a common side effect of RTX in autoimmune disease. Rigour has been reported in patients with advanced-stage follicular lymphomas under RTX treatment and might be related to the release of cytokines. ${ }^{39} 40$ This needs further analysis in forthcoming trials. In general, the use of RTX in the other systemic rheumatic diseases has shown good safety profile. A major concern associated with a long-term RTX use is progressive multifocal leukoencephalopathy. This was not observed in our short-term study, but longer follow-up in a larger number of patients and comparison with a placebo group is needed to establish a side effect profile specific for SSc. ${ }^{3641}$

Our study has certain limitations: First, observational trials can be confounded by indication. This accounts for the fact that in observational trials patients with higher need for treatment are more likely to get the medication of interest. However, we accounted for this potential confounder by matching patients for

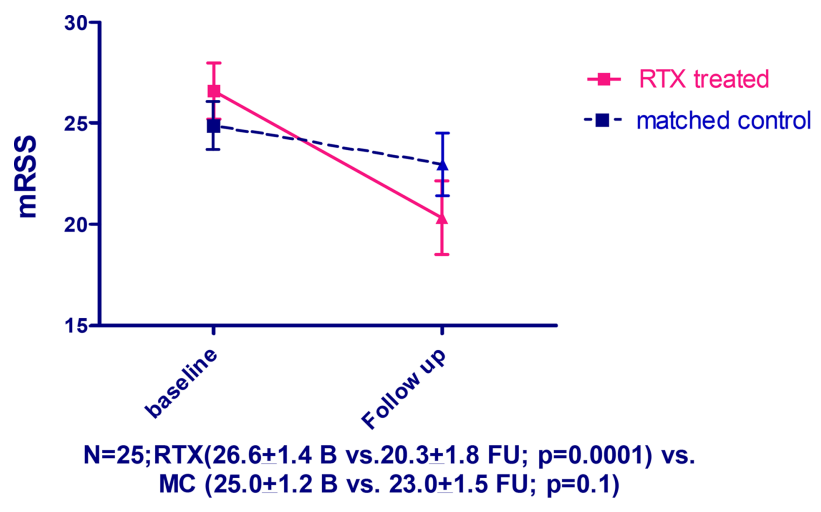

$\mathbf{A}$

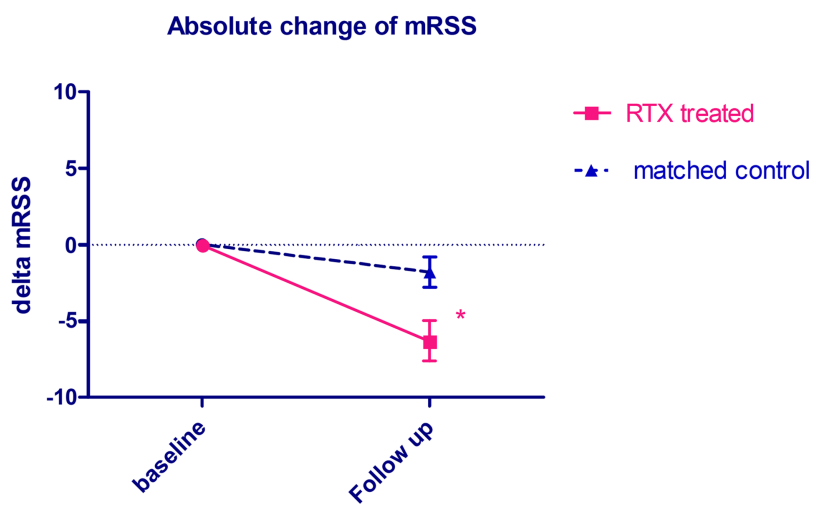

$N=25 ;-6.3+1.4$ vs. $-1.9+1.0 ; p=0.02$

B

Percental change of mRSS

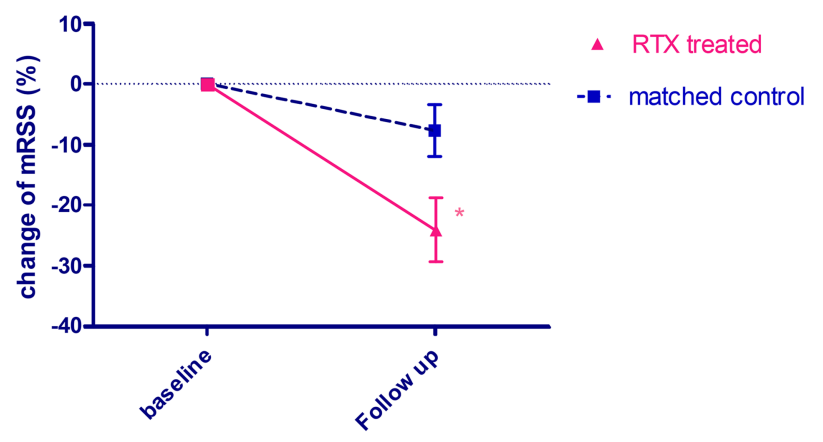

$\mathrm{N}=25 ;-24.0+5.2 \%$ vs. $-7.7+4.3 \% ; p=0.03$

C

Figure 2 (A) Modified Rodnan Skin Score (mRSS) at baseline and follow-up in systemic sclerosis (SSc) patients treated with rituximab (RTX) and matched-control SSc patients; B, baseline; FU, follow-up; MC, matched control. (B) Absolute change ( $\delta$ ) of mRSS at follow-up in RTX treated versus matched-control group of SSc patients. (C) Percental change of mRSS at follow-up in RTX treated versus Matched-control group of SSC patients.

major factors that influence the treatment decision for skin and lung, including severity of mRSS, disease duration and scleroderma subtype. While immunosuppressive co-treatment was matched between RTX patients and controls, we cannot exclude that the application of methylprednisolone during RTX applications had effects on skin and lung fibrosis, although this is unlikely with only one or two applications. When we compared patients with coapplication of methylprednisolone with those 


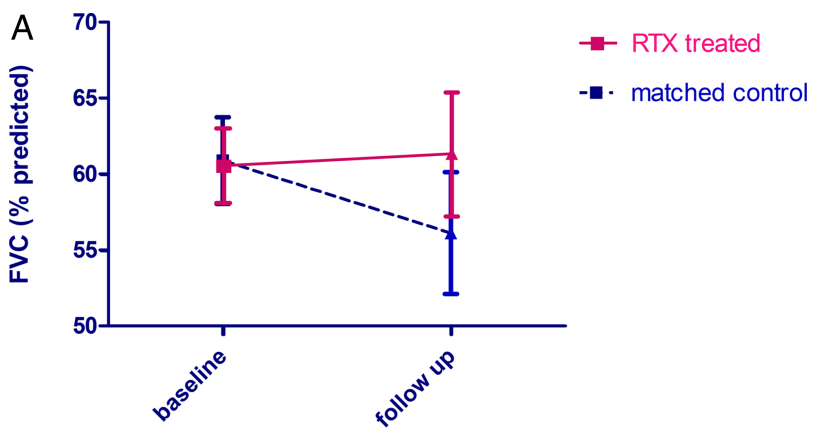

$\mathrm{N}=9$; RTX $(60.6+2.4$ B vs. $61.3+4.1 \mathrm{FU} ; \mathrm{p}=0.5)$ vs. MC $(60.9+2.8$ B vs. $56.1+4.0 \mathrm{FU} ; \mathrm{p}=0.02)$

B Absolute change of FVC (\% predicted)

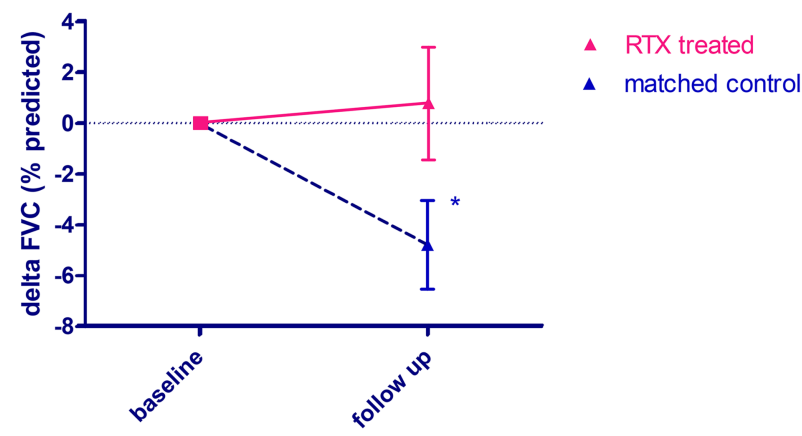

$\mathrm{N}=9 ; 0.8+2.2$ vs. $-4.8+1.7 ; p=0.01$

Figure 3 (A) Forced vital capacity (FVC) (\% predicted) at baseline and follow-up in the systemic sclerosis (SSc) patients with lung fibrosis treated with rituximab (RTX) versus matched-control SSc patients; $\mathrm{B}$, baseline; FU, follow-up; MC, matched control. (B) Absolute change of FVC (\% predicted).

without, the mRSS at follow-up did not differ significantly between the two groups (data not shown). In addition, history of previous immunosuppressive treatment longer than 3 months before RTX was not available in this study for both groups. Next, the mRSS, which was the measure of skin fibrosis in this study, shows a considerable inter-rater variability and also varies between experienced and less experienced examiners. However, in the EUSTAR network, centres are requested that the same examiner performs baseline and follow-up examinations. Furthermore, EUSTAR experts are trained regularly at EUSTAR courses, which have been shown to improve standardisation, intraobserver variability and within-patient SD values of the mRSS. ${ }^{33} 42$ Another potential limitation was that some of the data for this study had to be collected retrospectively, because they were not captured in the otherwise prospectively collected EUSTAR database. Finally, it has to be strongly emphasised that this is not a randomised controlled trial which allows definite conclusions on the efficacy of RTX on skin and lung fibrosis in SSc and the relative safety compared with placebo treatment. Randomisation and blinded treatment are an important part of clinical study design in SSc, and these issues need to be addressed in prospective, randomised, double-blinded, placebo-controlled phase III trials. Nevertheless, our study as the largest and controlled study available so far adds important aspects to RTX and $\mathrm{B}$ cell targeting in general as a potential antifibrotic treatment strategy in SSc. It provides the motivation for initiation of a phase III prospective, randomised, double-blinded, placebocontrolled trial in SSc, which is now the next necessary step to prove efficacy of B cell targeting on fibrotic manifestations in SSc.
Taken together, in this large, multicentre, observational trial using the EUSTAR database, we could show beneficial effects of RTX on skin and lung fibrosis in patients with SSc compared with matched-control SSc patients without RTX treatment. These promising results need to be confirmed in phase III randomised-controlled trials.

Collaborators Coauthors of the EUSTAR Rituximab study group: Paolo Airo: UO Rheumatology and Clinical Immunology, Spedali Civili, Brescia, Italy; Juan José Alegre Sancho: Hospital U Dr Peset, Valencia, Spain; Lidia Ananjeva: Institute of Rheumatology, Russian Academy of Medical Science, Moscow, Russia; Codrina Michaela Ancuta: Rheumatology Department, 'Gr. T. Popa' University of Medicine and Pharmacy, Clinical Rehabilitation Hospital, lasi, Romania; Martin Aringer: Rheumatology Department, University Hospital Dresden, Germany; Alexandra Balbir-Gurman: B. Shine Rheumatology Unit, Rambam Health Care Campus, Haifa, Israel; Francesco Paolo Cantatore: Rheumatology Unit, University of Foggia, Foggia, Italy; Paola Caramaschi: Rheumatology Unit, AOUI di Verona, Verona, Italy; Emmanuel Chatelus: Department of Rheumatology, University Hospital of Strasbourg, France; Veronica Codullo: Rheumatology Unit, Fondazione IRCCS Policlinico San Matteo and University of Pavia, Italy; Dominique Farge-Bancel: Paris-7 University Denis Diderot, Internal Medicine and Vascular Disease Unit, Inserm U976, Hopital Saint-Louis, AP-HP, Paris, France; Rosario Foti: Rheumatology Unit, A.O.U., Policlinico Vittorio Emanuele, Catania, Italy; Armando Gabrielli: Dipartimento di Scienze Cliniche e Molecolari, Clinica Medica, Università Politecnica delle Marche, Ancona, Italy; Jörg Henes: Department of Rheumatology, University Hospital Tübingen, Germany; Ilka Herrgott: Department of Dermatology, University of Münster, Germany; Florenzo lannone: Rheumatology Unit-DiM, School of Medicine, University of Bari, Italy; Francesca Ingegnoli: Division of Rheumatology, Department of Clinical Sciences \& Community Health, University of Milan, Italy; Esthela Loyo: Reumatologia e Inmunologia Clinica, Hospital Regional Universitario Jose Ma Cabral y Baez, Santiago, Dominican Republic; Marco Matucci-Cerinić: Department of Biomedicine, Division of Rheumatology AOUC \& Department of Experimental and Clinical Medicine, School of Medicine, University of Florence, Italy; Walid Ahmed Abdel Atty Mohamed: Unit of Rheumatology, Alexandria University, Egypt; Ulf Müller-Ladner: Department of Rheumatology and Clinical Immunology, Kerckhoff Clinic, Justus-Liebig University Giessen, Germany; Øyvind Palm: Department of Rheumatology, Oslo University Hospital Rikshospitalet, Oslo, Norway; Sergiu Popa: Department of Rheumatology, Republican Clinical Hospital, Chisinau, Republic of Moldova; Gabriela Riemekasten: Department of Rheumatology and Clinical Immunology, University Hospital Charité; German Rheumatism Research Centre (DRFZ) Berlin; Leibniz-Institute, Berlin, Germany; Simona Rednic: Rheumatology Clinic and 'Iuliu Hatieganu' University of Medicine and Pharmacy, Cluj-Napoca, Romania; Edoardo Rosato: Department of Clinical Medicine-Clinical Immunology Unit, Sapienza, University of Rome, Italy; Marta Saracco: Division of Rheumatology 'Ordine Mauriziano' Hospital, Torino, Italy; Agneta Scheja: Department of Clinical Sciences Lund, Section of Rheumatology, Lund University, Sweden; Vanessa Smith: Department of Rheumatology, Ghent University Hospital, Belgium; Carina Mihai: Department of Internal Medicine and Rheumatology Clinic, 'Ion Cantacuzino' Clinical Hospital, Bucharest, Romania; Gabriela Szucs: Institute of Medicine, Department of Rheumatology, University of Debrecen, Hungary; Matija Tomšič: Department of Rheumatology, University Medical Centre, Ljubljana, Slovenia; Gabriele Valentini: Rheumatology Unit, Second University of Naples, Naples, Italy; Ulrich A Walker: Department of Rheumatology, University Hospital Basel, Basel, Switzerland; Rene Westhovens: Skeletal Biology and Engineering Research Center, Department of Development and Regeneration KU Leuven; Rheumatology, University Hospitals Leuven, Belgium; Sule Kurhan Yavuz: Department of Rheumatology, University of Marmara, Istanbul, Turkey; Thierry Zenone: Department of Medicine, Unit of Internal Medicine, Valence, France.

Competing interests JHW Distler has consultancy relationships and/or has received research funding from Boehringer Ingelheim, Celgene, Bayer Pharma, Actelion, Pfizer, Ergonex, BMS, JB Therapeutics, Anaphore, Inc, Sanofi-Aventis, Novartis, Array Biopharma and Active Biotec in the area of potential treatments of scleroderma and is stock owner of 4D Science. O Distler has/had consultancy relationship and/or has received research funding in the area of systemic sclerosis and related conditions from Actelion, Pfizer, Ergonex, BMS, Sanofi-Aventis, United BioSource Corporation, Roche/Genentech, Medac, Biovitrium, Boehringer Ingelheim Pharma, Novartis, 4 D Science, Active Biotec, Bayer-Schering, Sinoxa, Serodapharm and EpiPharm. Y Allanore has consultancy relationships and/or has received research funding from Bayer Pharma, Actelion, Pfizer, Sanofi-Aventis and Roche, in the area of potential treatments of scleroderma. JMv Laar has received research support from Roche, Genentech (a Roche company), Trubion, and honoraria from Roche, Genentech, Pfizer, Abbott and Menarini. C Ancuta has/had consultancy relationship and/or received honoraria from Abbott, Pfizer, Roche/Genentech, Servier, Alfa Wasserman and Teva Pharmaceuticals. J Henes has received honoraria from Pfizer, Roche, and Abbvie. F lannone has/had consultancy relationship and/or has received research funding in the area of systemic sclerosis from Actelion. JJA Sancho has/had 
consultancy relationship and/or received honoraria from Actelion, Pfizer, Abbvie, BMS, Roche, UCB Pharma and MSD. V Smith has/had consultancy relationship and/ or has received research funding/support in the area of systemic sclerosis and related conditions from Actelion and Roche. UA Walker has/had consultancy relationship and/or has received research funding/support in the area of autoimmune diseases from Actelion and Roche. R Westhovens received research grants for his institution from Roche and UCB. T Zenone has received honoraria from Actelion.

\section{Funding None.}

Patient consent Obtained.

Ethics approval Ethics Committee Zurich, Switzerland.

Provenance and peer review Not commissioned; externally peer reviewed.

\section{REFERENCES}

1 Lafyatis $\mathrm{R}$, Kissin $\mathrm{E}$, York $\mathrm{M}$, et al. B cell depletion with rituximab in patients with diffuse cutaneous systemic sclerosis. Arthritis Rheum 2009;60:578-83.

2 Lafyatis R, O'Hara C, Feghali-Bostwick CA, et al. B cell infiltration in systemic sclerosis-associated interstitial lung disease. Arthritis Rheum 2007;56:3167-8.

3 Whitfield ML, Finlay DR, Murray Jl, et al. Systemic and cell type-specific gene expression patterns in scleroderma skin. Proc Natl Acad Sci USA 2003;100:12319-24.

4 Tedder TF, Inaoki M, Sato S. The CD19-CD21 complex regulates signal transduction thresholds governing humoral immunity and autoimmunity. Immunity 1997:6:107-18.

5 Fujimoto M, Sato S. B cell signaling and autoimmune diseases: CD19/CD22 loop as a B cell signaling device to regulate the balance of autoimmunity. J Dermatol Sci 2007:46:1-9.

6 Sato S, Hasegawa M, Fujimoto M, et al. Quantitative genetic variation in CD19 expression correlates with autoimmunity. J Immunol 2000;165:6635-43.

7 Hitomi Y, Tsuchiya N, Hasegawa M, et al. Association of CD22 gene polymorphism with susceptibility to limited cutaneous systemic sclerosis. Tissue Antigens 2007;69:242-9.

8 Saito E, Fujimoto M, Hasegawa M, et al. CD19-dependent B lymphocyte signaling thresholds influence skin fibrosis and autoimmunity in the tight-skin mouse. J Clin Invest 2002:109:1453-62.

9 Asano N, Fujimoto M, Yazawa N, et al. B Lymphocyte signaling established by the CD19/CD22 loop regulates autoimmunity in the tight-skin mouse. Am J Pathol 2004; 165:641-50.

10 Sato S, Fujimoto M, Hasegawa $M$, et al. Altered blood B lymphocyte homeostasis in systemic sclerosis: expanded naive B cells and diminished but activated memory B cells. Arthritis Rheum 2004;50:1918-27.

11 Matsushita T, Hasegawa M, Yanaba K, et al. Elevated serum BAFF levels in patients with systemic sclerosis: enhanced BAFF signaling in systemic sclerosis B lymphocytes. Arthritis Rheum 2006;54:192-201.

12 Matsushita T, Fujimoto M, Hasegawa M, et al. BAFF antagonist attenuates the development of skin fibrosis in tight-skin mice. J Invest Dermatol 2007;127:2772-80

13 Wynn TA. Fibrotic disease and the $\mathrm{T}(\mathrm{H}) 1 / \mathrm{T}(\mathrm{H}) 2$ paradigm. Nat Rev Immunol 2004;4:583-94.

14 Snapper CM, Waegell W, Beernink H, et al. Transforming growth factor-beta 1 is required for secretion of $\lg G$ of all subclasses by LPS-activated murine $B$ cells in vitro. J Immunol 1993;151:4625-36.

15 Ihn H, Yamane K, Kubo M, et al. Blockade of endogenous transforming growth factor beta signaling prevents up-regulated collagen synthesis in scleroderma fibroblasts: association with increased expression of transforming growth factor beta receptors. Arthritis Rheum 2001;44:474-80.

16 Hasegawa M, Hamaguchi Y, Yanaba K, et al. B-lymphocyte depletion reduces skin fibrosis and autoimmunity in the tight-skin mouse model for systemic sclerosis. Am J Pathol 2006;169:954-66.

17 Perosa F, Prete M, Racanelli V, et al. CD20-depleting therapy in autoimmune diseases: from basic research to the clinic. J Intern Med 2010;267:260-77.

18 McGonagle D, Tan AL, Madden J, et al. Successful treatment of resistant scleroderma-associated interstitial lung disease with rituximab. Rheumatology (Oxford) 2008:47:552-3.

19 Daoussis D, Liossis SN, Tsamandas AC, et al. Is there a role for B-cell depletion as therapy for scleroderma? A case report and review of the literature. Semin Arthritis Rheum 2010;40:127-36.
20 Yoo WH. Successful treatment of steroid and cyclophosphamide-resistant diffuse scleroderma-associated interstitial lung disease with rituximab. Rheumatol Int 2012;32:795-98.

21 Daoussis $D$, Liossis SN, Tsamandas AC, et al. Experience with rituximab in scleroderma: results from a 1-year, proof-of-principle study. Rheumatology (Oxford) 2010:49:271-80

22 Bosello S, De Santis M, Lama G, et al. B cell depletion in diffuse progressive systemic sclerosis: safety, skin score modification and IL-6 modulation in an up to thirty-six months follow-up open-label trial. Arthritis Res Ther 2010;12:R54.

23 Smith V, Van Praet JT, Vandooren B, et al. Rituximab in diffuse cutaneous systemic sclerosis: an open-label clinical and histopathological study. Ann Rheum Dis 2010;69:193-7.

24 Smith V, Piette Y, van Praet JT, et al. Two-year results of an open pilot study of a 2-treatment course with rituximab in patients with early systemic sclerosis with diffuse skin involvement. J Rheumatol 2013;40:52-7.

25 Khanna D, Furst DE, Clements PJ, et al. Responsiveness of the SF-36 and the Health Assessment Questionnaire Disability Index in a systemic sclerosis clinical trial. J Rheumatol 2005;32:832-40.

26 Poole JL, Steen VD. The use of the Health Assessment Questionnaire (HAQ) to determine physical disability in systemic sclerosis. Arthritis Care Res 1991; 4:27-31.

27 Redelmeier DA, Lorig K. Assessing the clinical importance of symptomatic improvements. An illustration in rheumatology. Arch Intern Med 1993;153:1337-42.

28 Daoussis $\mathrm{D}$, Liossis SN, Tsamandas AC, et al. Effect of long-term treatment with rituximab on pulmonary function and skin fibrosis in patients with diffuse systemic sclerosis. Clin Exp Rheumatol 2012;30:S17-22.

29 Meier FM, Frommer KW, Dinser R, et al. Update on the profile of the EUSTAR cohort: an analysis of the EULAR Scleroderma Trials and Research group database. Ann Rheum Dis 2012;71:1355-60.

30 Walker UA, Tyndall A, Czirjak L, et al. Clinical risk assessment of organ manifestations in systemic sclerosis: a report from the EULAR Scleroderma Trials And Research group database. Ann Rheum Dis 2007;66:754-63.

31 Clements $\mathrm{P}$, Lachenbruch $\mathrm{P}$, Siebold J, et al. Inter and intraobserver variability of total skin thickness score (modified Rodnan TSS) in systemic sclerosis. I Rheumatol 1995;22:1281-5.

32 Ionescu R, Rednic S, Damjanov N, et al. Repeated teaching courses of the modified Rodnan skin score in systemic sclerosis. Clin Exp Rheumatol 2010;28:S37-41.

33 Czirjak L, Foeldvari I, Muller-Ladner U. Skin involvement in systemic sclerosis. Rheumatology (Oxford) 2008;47(Suppl 5):v44-5.

34 Masi AT. Preliminary criteria for the classification of systemic sclerosis (scleroderma). Subcommittee for scleroderma criteria of the American Rheumatism Association Diagnostic and Therapeutic Criteria Committee. Arthritis Rheum. 1980;23:581-90.

35 Khanna $D$, Furst $D E$, Hays RD, et al. Minimally important difference in diffuse systemic sclerosis: results from the D-penicillamine study. Ann Rheum Dis 2006;65:1325-9.

36 Edwards JC, Szczepanski L, Szechinski J, et al. Efficacy of B-cell-targeted therapy with rituximab in patients with rheumatoid arthritis. N Eng/ I Med 2004;350:2572-81.

37 Emery P, Fleischmann R, Filipowicz-Sosnowska A, et al. The efficacy and safety of rituximab in patients with active rheumatoid arthritis despite methotrexate treatment: results of a phase IIB randomized, double-blind, placebo-controlled, dose-ranging trial. Arthritis Rheum 2006;54:1390-400.

38 Cohen SB, Emery P, Greenwald MW, et al. Rituximab for rheumatoid arthritis refractory to anti-tumor necrosis factor therapy: results of a multicenter, randomized, double-blind, placebo-controlled, phase III trial evaluating primary efficacy and safety at twenty-four weeks. Arthritis Rheum 2006;54:2793-806.

39 Feuring-Buske M, Kneba M, Unterhalt M, et al. IDEC-C2B8 (Rituximab) anti-CD20 antibody treatment in relapsed advanced-stage follicular lymphomas: results of a phase-II study of the German Low-Grade Lymphoma Study Group. Ann Hematol 2000;79:493-500.

40 Boye J, Elter T, Engert A. An overview of the current clinical use of the anti-CD20 monoclonal antibody rituximab. Ann Oncol 2003;14:520-35.

41 Calabrese LH, Molloy ES. Progressive multifocal leucoencephalopathy in the rheumatic diseases: assessing the risks of biological immunosuppressive therapies. Ann Rheum Dis 2008;67(Suppl 3):iii64-5.

42 Czirjak L, Nagy Z, Aringer M, et al. The EUSTAR model for teaching and implementing the modified Rodnan skin score in systemic sclerosis. Ann Rheum Dis 2007;66:966-9. 